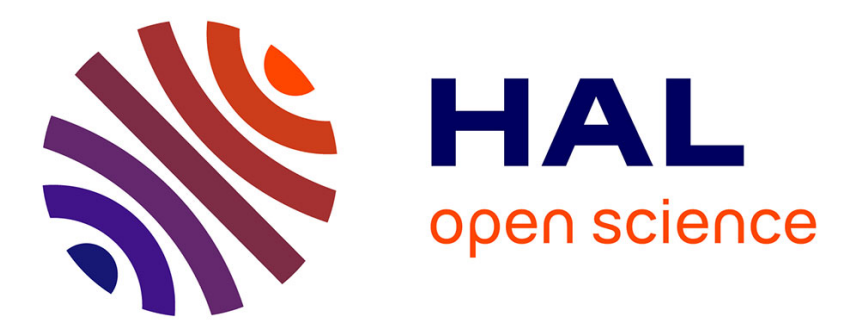

\title{
Airbus/future of aircraft factory HRP-2 as universal worker proof of concept
}

Olivier Stasse, Andreas Orthey, Francesco Morsillo, Mathieu Geisert, Nicolas Mansard, Maximilien Naveau, Christian Vassallo

\section{To cite this version:}

Olivier Stasse, Andreas Orthey, Francesco Morsillo, Mathieu Geisert, Nicolas Mansard, et al.. Airbus/future of aircraft factory HRP-2 as universal worker proof of concept. International Conference on Humanoid Robotics, Nov 2014, Madrid, Spain. 10.1109/HUMANOIDS.2014.7041488 hal-01122476

\section{HAL Id: hal-01122476 https://hal.science/hal-01122476}

Submitted on 4 Mar 2015

HAL is a multi-disciplinary open access archive for the deposit and dissemination of scientific research documents, whether they are published or not. The documents may come from teaching and research institutions in France or abroad, or from public or private research centers.
L'archive ouverte pluridisciplinaire HAL, est destinée au dépôt et à la diffusion de documents scientifiques de niveau recherche, publiés ou non, émanant des établissements d'enseignement et de recherche français ou étrangers, des laboratoires publics ou privés. 


\title{
Airbus/Future of Aircraft Factory HRP-2 as Universal Worker Proof of Concept.
}

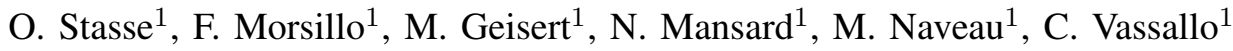

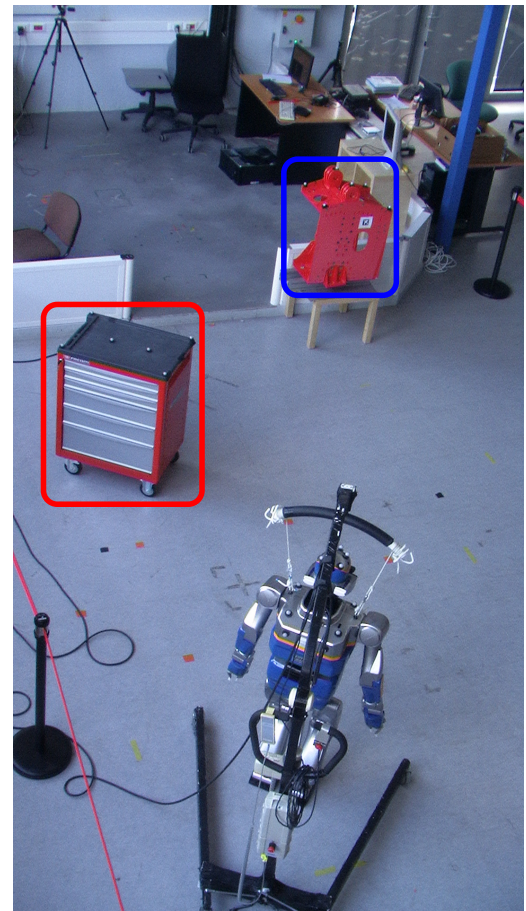

Fig. 1. Situation of the experiment: The robot has to go in the vicinity of the pylon engine depicted in blue while avoiding the red moving toolbox

\begin{abstract}
In this paper, we present a preliminary proof of concept (PoC) aiming at introducing humanoid robots in an aircraft factory. The PoC was aiming at demonstrating the capacity of HRP-2 to deal with three aspects needed in a factory: reactivity to change in the environment, visual feedback and on-line motion generation. The limits reached in this PoC are here highlighted to draw some direction of research focused on the needs of Aircraft manufacturers.

Humanoid robot technology has reached a high level of maturity and now has been implemented on several high quality robots such as Asimo, Atlas, TORO or HRP-2. They have been long envisionned as universal worker which can be used in factory, this video present a PoC in this context.
\end{abstract}

\section{FAST RE-PLANNING FOR MOVING OBSTACLE AVOIDANCE}

The setup of this scenario is depicted in Fig. 1. Using information provided by motion capture the robot is able to

\footnotetext{
*This work was supported by the Airbus/Future of Aircraft Factory.

${ }^{1}$ are with the CNRS, LAAS,7 av. du Colonel Roche, F-31400, Toulouse, France, Univ de Toulouse, LAAS, F-31400, Toulouse, France surname. namedlaas. fr
}

plan autonomously footsteps from its current location to the pylon engine. The pylon engine is the piece which connect the aircraft engine and the wing. This is where the robot has to perform his screwing motion. A human may move the toolbox such that it crosses the path of the robot. The robot is able to change its footsteps and avoid the toolbox.

The robot is searching over a set of pre-defined action set which are known to be feasible. The small foot-print of the action set allows for real-time planning search over a costfunction which includes a metric from the starting point to the robot current location and a metric from the robot current location to its final goal. This solution is currently based on the family of $\mathrm{A}^{*}$ algorithms as proposed in the following papers [1], [2], [3] with demonstrations on various humanoid robots such as ASIMO, HRP-2 or NAO. The method here is also based on our work published in [4]. More precisely, from a set of quasi static half-steps, the robot trajectory is speed up using an analytical pattern generator coupled with PQP to test if the trajectories are without collision.

The robot is able to plan very quickly a path from its current location to the final one as depicted in the bottom of the first sequence of the video. If the toolbox is put on the robot path and if the robot is three steps away it can avoid it. The three steps are a limitation related to the pattern generator which needs this information on the future. It is currently quite difficult to find in real-time a full wholebody trajectory which avoid obstacles and maintain the robot balance. The goal of this experiment showing the reactive capabilities of the system in this specific context has been reached.

A* approach are using a limited set of actions to simplify the problem solving. However in situation a bit more complex the robot tends to make unnecessary long sequence of steps because it is exploring only this limited sequence of actions. This was the main point of using a more aggressive approach proposed in [4]. In order to adapt more reactively the plan, the system would need a rather different control system for balancing. This is the subject of the second experience.

\section{REACTIVE WALKING PATTERN GENERATOR}

The balance control law of the robot takes as an input a velocity reference, and the system try to find footsteps such that the robot Center-Of-Mass is following as much as it can the velocity reference. In this PoC, two ways were tried to compute a reference velocity: visual servoing, and Euclidian distance between the object pose and the robot pose using a Motion Capture system. A more detailed description of 
the balance control law developed in the context of the French Research Project R-Blink is available in [5]. A first experiment with this setup was realized in [6].

In the video the robot is able to follow the position of the engine pylon given by the motion capture system in the frontal plan. This library was successfully used for the experiments described in [6]. It was interesting to test it on a different HRP-2 to check the portability of the software.

Unfortunately if the ViSP library has been working quite well with the wooden mockup made by LAAS of the engine pylon, (as shown on the third section of the section) it did failed with the 3D-print provided by Airbus. The main reason is the lack of sharp edge in the back of the pylon. We tried various strategies such as including a Kalman Filter, introducing knowledge in the tracker, but it turns out to be easier to use the Motion Capture System. If this control law shows great promises, it needs further improvement in the robustness part to be used in a repeatable setup. Our current line of research is to improve the balance control by including resolved momentum control, and dynamic filter.

\section{WHOLE BODY MOTION FOR SCREWING}

The goal of this third behavior was to check whether or not if the humanoid robot HRP-2 is able to make the basic motion necessary to go towards positions allowing a screwing action on the engine pylon. Note that the robot assume that an extender of $10 \mathrm{~cm}$ is put at the extremity of the electric screwdriver.

The behavior realized was based on the stack of tasks [7] a framework which is combining different control laws together and takes advantage of humanoid robots redundancy. Its software implementation has been used since 2006 to implement various demonstrators. The goal of the mathematical formulation is to enforce properties which make the control safer by checking strictly some limits, and still make possible to change dynamically the control and being efficient [8].

In the frame of the $\mathrm{PoC}$, the main point was to test the work space of the HRP-2 humanoid with a 3D print of an AIRBUS screwdriver. The robot was able to reach most of the positions in the frontal plane of the engine pylon. On the wood mockup we have been able to realize a behavior where the robot is visually tracking the point by a whole body motion without moving the feet. However the vision process did not work properly on the 3D-print, so we decide to switch to the Motion Capture instead. This is demonstrated in the third and fourth sequence of the video. It has to be notice that the transition between the points is not formally proved or checked. It worked because the robot is highly redundant and we did not push the robot to the limit. Trying to reach the screws at the top of the engine pylon will need probably planning trajectories. During the process we tested our control in acceleration to improve the behavior of the system.

The behavior was very much improved but we found one problem in our solver when the task is a posture. For this reason we came back to the usual kinematic control.
In addition we have tested very high gain showing that the robot is able to go up to $1.6 \mathrm{~s}$ between the transitions. But the momentum involved by this fast motion has to be taken into account at a higher level and cannot be recovered by the current robot stabilizer. For this reason, we kept a rather low gain for the robot and we are currently in the process of improving it. Finally we noted that when the robot is lowering down, it is close to self-collision. This can be fixed using self-collision avoidance, but call for a deep interaction between planning and control. This is especially true when using vision. A slight drift in rotation may prevent the convergence of the controller to a screwing point.

\section{CONCLUSION}

The robot HRP-2 is able to evolve in an environment with moving objects such as a toolbox pushed by a human. We have shown that using a localization system, it is possible to walk but also to make the robot perform whole body motion with one control architecture. The current limitation of the system is the complexity of the environment and the large space of possible motion for the robot. Still for the current setup on which we agree for the PoC, the current techniques seem applicable. It is however possible to improve the speed of the robot, and try the next level where the robot is really trying to perform the screwing action. We are looking forward to continue this line of action and try more challenging environment, and motion.

\section{ACKNOWLEDGEMENT}

We gratefully acknowledge S. Boria and B. Duprieux from Airbus/Future of the Aircraft Factory for their help and support.

\section{REFERENCES}

[1] J. Chestnutt, Motion Planning for Humanoid Robots. Springer, 2010, ch. Chapter 1 - Navigation and gait planning, pp. 1-30.

[2] S. K. J. Kuffner and N. Nishiwaki, "A* for real-time foot-step planning," in IEEE/RAS Int. Conf. on Humanoid Robotics (ICHR), 2002.

[3] A. Hornung, A. Dornbush, M. Likhachev, and M. Bennewitz, "Anytime search-based footstep planning with suboptimality bounds," in IEEE/RAS Int. Conf. on Humanoid Robotics (ICHR), 2012.

[4] N. Perrin, O. Stasse, L. Baudouin, F. Lamiraux, and E. Yoshida, "Fast humanoid robot collision-free footstep planning using swept volume approximations," IEEE Transactions on Robotics, vol. 28, no. 2, pp. 427-439, 2012.

[5] A. Herdt, N. Perrin, and P.-B. Wieber, "Walking without thinking about it," in IEEE International Conference on Robot Systems (IROS), 2010, pp. 190-195.

[6] C. Dune, A. Herdt, E. Marchand, O. Stasse, P.-B. Wieber, and E. Yoshida, "Vision based control for humanoid robots," in IEEE/RAS International Conference on Intelligent Robot and Systems (IROS), Workshop on Visual Control of Mobile Robots (ViCoMor), 2011.

[7] N. Mansard, O. Stasse, P. Evrard, and A. Kheddar, "A versatile generalized inverted kinematics implementation for collaborative working humanoid robots: The stack of tasks," in International Conference on Advanced Robotics (ICAR), June 2009, p. 119.

[8] A. Escande, N. Mansard, and P.-B. Wieber, "Hierarchical quadratic programming." International Journal of Robotics Research, Februrary 2014. 